\title{
A Semantic Foundation for Hidden State
}

\author{
Jan Schwinghammer ${ }^{1}$, Hongseok Yang ${ }^{2}$, Lars Birkedal $^{3}$, \\ François Pottier ${ }^{4}$, and Bernhard Reus ${ }^{5}$ \\ 1 Saarland Univ. \\ 2 Queen Mary Univ. of London \\ ${ }^{3}$ IT Univ. of Copenhagen \\ 4 INRIA \\ 5 Univ. of Sussex
}

\begin{abstract}
We present the first complete soundness proof of the antiframe rule, a recently proposed proof rule for capturing information hiding in the presence of higher-order store. Our proof involves solving a non-trivial recursive domain equation, and it helps identify some of the key ingredients for soundness.
\end{abstract}

\section{Introduction}

Information hiding, or hidden state, is one of the key design principles used by programmers in order to control the complexity of large-scale software systems. Being able to exploit this principle in the formal setting of a program logic could represent an important step towards the development of modular, scalable program verification techniques.

The idea is that an object (or function, or module) need not reveal in its interface the fact that it owns and maintains a private, mutable data structure. Hiding this internal invariant from the client has several beneficial effects. First, the complexity of the object's specification is slightly decreased. More importantly, the client is relieved from the need to thread the object's invariant through its own code. In particular, when an object has multiple clients, they are freed from the need to cooperate with one another in threading this invariant. Last, by hiding its internal state, the object escapes the restrictions on aliasing and ownership that are normally imposed to objects with mutable state.

It is worth emphasizing that hiding and abstraction (as studied, for instance, in separation logic [-3]) are distinct mechanisms, which may co-exist within a single program logic 1

The recently proposed anti-frame proof rule [4] enables hiding in the presence of higher-order store (i.e., memory cells containing procedures or code fragments). In this paper, we study the semantic foundation of the anti-frame rule,

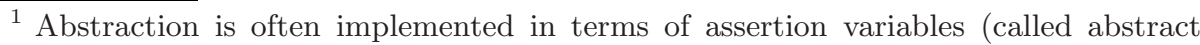
predicates by Parkinson) that describe the private data structures of an object. These variables are exposed to a client, but their definitions are not, so that the object's internals are presented to the client in an abstract form. Hiding, on the other hand, conceals the object's internals completely.
}

L. Ong (Ed.): FOSSACS 2010, LNCS 6014, pp. 2 17, 2010.
(c) Springer-Verlag Berlin Heidelberg 2010 
and give the first complete soundness proof for it. Our proof involves the solution of an intricate recursive domain equation, and it helps identify some of the key ingredients for soundness.

Information hiding with frame and anti-frame rules. Our results push the frontier of recent logic-based approaches to information hiding. These approaches adopt a standard semantics of the programming language, and deal with information hiding on a logical basis, by extending a Hoare calculus with special proof rules. These usually take the form of frame rules that allow the implementation of an object to ignore (hence implicitly preserve) some of the invariants provided by the context, and of anti-frame rules, which allow an object to hide its internal invariant from the context [4-77].

In its simplest form, the frame rule [5] states that invariants $R$ can be added to valid triples: if $\{P\} C\{Q\}$ is valid, then so is $\{P * R\} C\{Q * R\}$, where the separating conjunction $P * R$ indicates that $P$ and $R$ govern disjoint regions of the heap. In subsequent developments, the rule was extended to handle higherorder procedures [6, 7] and higher-order store [8, 9]. Moreover, it was argued that both extensions of the rule support information hiding: they allow one to hide the invariant of a module [6] and to prove properties of clients, as long as the module is understood in continuation-passing style.

Thorough semantic analyses were required to determine the conditions under which these extensions of the frame rule are sound. Indeed, the soundness of these rules raises subtle issues. For instance, the frame rule for higher-order procedures turns out to be inconsistent with the conjunction rule, a standard rule of Hoare logic 6, 7]. Furthermore, seemingly innocent variants of the frame rule for higher-order store have been shown unsound [9, 10].

In the most recent development in this line of research, Pottier [4] proposed an anti-frame rule, which expresses the information hiding aspect of an object directly, instead of in continuation-passing style. Besides giving several extensive examples of how the anti-frame rule supports hidden state, Pottier argued that the anti-frame rule is sound by sketching a plausible syntactic argument. This argument, however, relied on several non-trivial assumptions about the existence of certain recursively defined types and recursively defined operations over types.

In this paper, we systematically study the semantic foundation of hidden state, as captured by frame and anti-frame rules, in the presence of higher-order store. In particular, we describe our soundness proof of a program logic that has both frame and anti-frame rules.

Overview of the technical development. The anti-frame rule was originally proposed in an expressive type system for an ML-like language [4]. In the context of separation logic, it becomes an inference rule for deriving Hoare triples. A slightly simplified version of it takes the following form:

$$
\frac{\{P \otimes R\} C\{(Q \otimes R) * R\}}{\{P\} C\{Q\}}
$$


Recall that the separating conjunction $P^{\prime} * Q^{\prime}$ holds of a heap when the heap can be split into two sub-heaps that respectively satisfy $P^{\prime}$ and $Q^{\prime}$. In order to specify properties of stored code, we allow assertions to contain nested triples [9], and introduce a $\otimes$ operator, whose meaning is roughly the following: $P^{\prime} \otimes R^{\prime}$ denotes a version of $P^{\prime}$ where $R^{\prime}$ has been $*$-conjoined with the pre- and post-conditions of every triple, including deeply nested triples.

In the anti-frame rule above, the code $C$ can be thought of as allocating and initializing an object. The assertion $R$ describes an internal invariant of this object, which one wishes to hide. The conjunct $-* R$ in the post-condition of the premise ensures that the invariant $R$ is established by $C$, so $R$ holds initially. The two occurrences of $-\otimes R$ in the premise guarantee that every triple that appears in the premise has $R$ as pre- and post-conditions, so every interaction between the object and the outside preserves $R$. The invariant $R$ does not appear in the conclusion of the rule, so one reasons about the rest of the program just as if the object had no internal state.

Our soundness proof of the anti-frame rule is based on two key components. The first is a new interpretation of Hoare triples, which explicates the universal and existential quantifications that are implicit in the anti-frame rule. Let $P \circ R$ abbreviate $(P \otimes R) * R$. Roughly speaking, in our interpretation, a triple $\{P\} C\{Q\}$ is valid if, for all invariants $R$, the triple

$$
\{P \circ R\} C\left\{\exists R^{\prime} . Q \circ\left(R \circ R^{\prime}\right)\right\}
$$

holds in the standard interpretation of triples. Pottier [4] showed how the antiframe rule allows encoding ML-like weak references in terms of strong references. Readers who are familiar with models of ML references (see, e.g., 11]) may thus find the above interpretation natural. Roughly speaking, the code $C$ has a function type $P \rightarrow Q$, whose interpretation is: "for all worlds $R$, if $C$ is given an argument of type $P$ in world $R$, then, for some future world $R \circ R^{\prime}$ (an extension of $R$ ), $C$ returns a result of type $Q$ in world $R \circ R^{\prime}$."

The second element in our soundness proof is a formalization of the above intuition. Our interpretation of assertions is parameterized by a set $W$ of worlds, or invariants, so that, semantically, an assertion is a function $W \rightarrow \mathcal{P}($ Heap $)$ from worlds to (certain) sets of heaps. Corresponding to $R \circ R^{\prime}$ in (1), there is a semantic operation $\circ$ on $W$ that lets us combine two invariants. This operation induces a preorder on invariants, whereby $R \circ R^{\prime}$ is greater than (i.e., a future world of) $R$.

In order to prove that the anti-frame rule is sound, we require assertions $P$ to be monotonic with respect to the preorder on invariants: that is, $P(R)$ must be a subset of $P\left(R \circ R^{\prime}\right)$, for all $P, R$ and $R^{\prime}$. This lets us relate an assertion $P$ at two different invariants $R_{0}$ and $R_{1}$, by first exhibiting an upper bound $R_{2}$ of $R_{0}$ and $R_{1}$ and then using the monotonicity to conclude $P\left(R_{i}\right) \subseteq P\left(R_{2}\right)$ for $i \in\{0,1\}$. This forms an important step of our soundness proof.

In order to present our soundness proof as abstractly and elegantly as we can, we begin with an axiomatization of worlds and world composition, that is, we state a number of requirements that worlds should satisfy (Section 2). This allows us to define the interpretation of triples and establish some of its key properties in an abstract setting (Section 3 ). 
In Sections 4 and 5 we move to a more concrete setting and present a small imperative programming language that features higher-order store, in the form of storable commands. We equip it with a proof system, which features nested Hoare triples, frame rules, and anti-frame rules. As in Pottier's original setting, in this system, it is desirable that every assertion $R$ be allowed to play the role of an invariant. As a consequence, in this concrete instance, the set of invariants $W$ should be isomorphic to the semantic domain of assertions.

In summary, for this instance the requirements that we have described above amount to the following non-standard recursive domain equation:

$$
\text { Assert } \cong \text { Assert } \rightarrow_{m} \mathcal{P}(\text { Heap }) .
$$

The subscript $-{ }_{m}$ indicates that we consider only the subset of monotonic functions. By restricting the codomain to subsets of Heap that satisfy particular conditions, and by further restricting the function space, we can find a solution to (a variant of) equation (2) in a category of complete metric spaces. However, because monotonicity is defined in terms of the ordering over assertions, which itself is defined in terms of the operation of composition $\circ$ on $W$ (recall that $W$ and Assert are isomorphic), we cannot do this using "off-the-shelf" techniques for solving recursive domain equations, like those of Rutten [12] or Birkedal et al. [13]. Instead, we obtain a solution by explicitly constructing the inverse limit of an appropriately chosen sequence of approximations to (2). We discuss these challenges and our solution in more detail in Section 4 .

Contributions. In summary, our main contributions are the following:

- We highlight which semantic ingredients are critical in establishing the validity of the frame and anti-frame rules. We hope that this will lead to increased understanding, and expect that these ingredients can be used as building blocks in the soundness proofs of future logics with information hiding principles.

- We give a proof of the soundness of a program logic that includes frame and anti-frame rules for higher-order store.

For space reasons, many proofs are omitted; some can be found in the full version of this paper [14].

\section{Semantic Setup}

In this section, we describe semantic ingredients that can be used to validate (certain types of) anti-frame and frame rules.

Programming language. Our assumptions on the semantics of the programming language are fairly standard. We assume that there is a (pointed, chain-complete partially ordered) set of heaps, Heap, and that commands either diverge, terminate successfully, or fault, i.e., that Com $=$ Heap $\multimap\left(\right.$ Heap $\left.\oplus\{\text { error }\}_{\perp}\right)$ is the set of (strict continuous) functions into Heap with an error element adjoined. We also 
assume that there exists a family of projection functions $\left(\pi_{k}: \text { Heap } \multimap \text { Heap }\right)_{k \in \mathbb{N}}$. The images of these projection functions must contain only finite element 2 and the projection functions must satisfy the following conditions:

$-\perp=\pi_{0}(h) \sqsubseteq \ldots \sqsubseteq \pi_{k}(h) \sqsubseteq \pi_{k+1}(h) \sqsubseteq \ldots \sqsubseteq h$ for all $h \in$ Heap, i.e., the $\pi_{k}$ 's form an increasing chain of approximations of the identity on Heap;

$-\pi_{j} \circ \pi_{k}=\pi_{\min \{j, k\}}$ for all $j, k$; in particular, every $\pi_{k}$ is idempotent;

- $\bigsqcup_{k} \pi_{k}(h)=h$, i.e., every heap is the limit of its approximations.

For example, these conditions hold if Heap is an SFP domain (e.g., [15]) with a particular choice of projections. Finally, we assume a partial commutative associative operation $h \cdot h^{\prime}$, which intuitively lets us combine heaps with disjoint locations and is compatible with the projections: $\pi_{k}\left(h \cdot h^{\prime}\right)=\pi_{k}(h) \cdot \pi_{k}\left(h^{\prime}\right)$.

We write $\mathbb{N}_{\infty}$ for the natural numbers extended with $\infty$, with $\infty+k=k+\infty=$ $\infty$. We define $\pi_{\infty}=i d$ and $2^{-\infty}=0$. The rank of $h$, written $r n k(h)$, is the least $k \in \mathbb{N}_{\infty}$ such that $\pi_{k}(h)=h$.

Uniformity and distance. Our program logics concern properties of heaps that are closed under the projection functions $\pi_{k}$. We write $U A d m$ for the set of admissible 3 subsets $p \subseteq$ Heap that are uniform: for any $k \in \mathbb{N}$, if $h \in p$ then $\pi_{k}(h) \in p$. Using the heap combination operation, we define separating conjunction $p * q$ for $p, q \in U A d m$ in the usual way: $h \in p * q$ iff $h=h_{1} \cdot h_{2}$ for some $h_{1} \in p$ and $h_{2} \in q$. We assume that $p * q$ is in $U A d m \square$

Uniformity gives rise to a notion of distance between (or, similarity of) properties of heaps. More precisely, writing $\pi_{k}(p)$ for the image of $p$ under the projection $\pi_{k}$, the function $d(p, q)=2^{-\sup \left\{k \in \mathbb{N}_{\infty} \mid \pi_{k}(p)=\pi_{k}(q)\right\}}$ defines a notion of distance on $U A d m$. This function satisfies the requirements of a 1-bounded $u l-$ trametric: that is, $d$ takes real values in the interval $[0,1]$, is symmetric, is such that $d(p, q)=0$ holds iff $p=q$, and satisfies $d(p, q) \leq \max \{d(p, r), d(r, q)\}$ for all $p, q, r \in U A d m$. With respect to this metric, UAdm is complete in the usual sense that every Cauchy sequence has a limit. The metric and this completeness result of $U A d m$ make it possible to model recursively defined assertions using the Banach fixed point theorem.

Worlds and assertions. Our semantics of assertions is defined in the category CBUlt of complete 1-bounded ultrametric spaces and non-expansive functions. This means that every semantic domain involved in the semantics has an appropriate notion of distance and that every function is non-expansive, i.e., the distance between two outputs is no greater than the distance between the two corresponding inputs.

The main ingredients necessary for validating forms of anti-frame and frame rules are a set of possible worlds, and an interpretation of the worlds as assertions. Thus, we require:

\footnotetext{
$\overline{{ }^{2} \text { An element }} d$ in a cpo $D$ is finite iff for all chains $\left\{d_{n}\right\}_{n \in \omega}$ in $D, d \sqsubseteq \bigsqcup_{n \in \omega} d_{n}$ implies that $d \sqsubseteq d_{n}$ for some $n$.

${ }^{3}$ The admissibility of $p$ means that $p$ is closed under limits of chains and contains $\perp$.

${ }^{4}$ This assumption holds when Heap is constructed in a standard way in terms of finite partial functions or records, just like our model in Sections 4 and 5.
} 
1. A monoid $(W, e, \circ)$ of worlds, or invariants, where $W$ is an object in CBUlt and the operation $\circ$ is non-expansive with respect to this metric. The monoid structure induces a preorder $\sqsubseteq$ on $W$, by $w \sqsubseteq w^{\prime} \Leftrightarrow \exists w_{0} \in W . w^{\prime}=w \circ$ $w_{0}$. Note that $\circ$ is in general not commutative, and that $w^{\prime}$ is obtained by extending $w$ on the right. Using this preorder, we define a domain

$$
\text { Assert } \stackrel{\text { def }}{=}\left(\frac{1}{2} \cdot W\right) \rightarrow_{m} U A d m .
$$

for assertions. Here, $\frac{1}{2} \cdot W$ denotes the scaling of the distance function on $W$ by $1 / 2$, and the function space consists of the non-expansive monotone functions. Assertions are thus parameterized by worlds, and this parameterization satisfies two conditions. The first condition is contractiveness, meaning that the distance between worlds gets reduced when they are used in an assertion: $d\left(p\left(w_{0}\right), p\left(w_{1}\right)\right) \leq d\left(w_{0}, w_{1}\right) / 2$ for all $p \in$ Assert and all $w_{0}, w_{1} \in W$. In the definition, contractiveness is formalized in two steps, first by scaling down the distance of worlds by $1 / 2$ and then by stipulating that assertions should preserve this scaled-down distance (i.e., be non-expansive).

The second condition is monotonicity: $p(w) \subseteq p\left(w \circ w_{0}\right)$ holds for all $p \in$ Assert and all $w, w_{0} \in W$. Here, $w_{0}$ can be thought of as an invariant that is hidden in world $w$ and revealed in world $w \circ w_{0}$. If some heap $h$ satisfies the assertion $p$ while $w_{0}$ is hidden, then $h$ still satisfies $p$ after $w_{0}$ is revealed. Intuitively, because the commands stored in the heap $h$ do not know about the invariant $w_{0}$, they must preserve it.

2. A non-expansive coercion function $i: W \rightarrow$ Assert, which offers a way of interpreting worlds as assertions.

We do not in general require $W \cong$ Assert: a one-way coercion is sufficient for our purposes. In fact, it is possible to define instances of our framework where $W$ is strictly "smaller" than Assert. Such a restriction, where not every assertion can play the role of a hidden invariant, can be exploited to establish the soundness of stronger versions of anti-frame and frame rules than the ones in the literature [4, 9]. For details, see Appendix D of the full version of this paper [14].

For the moment, we simply assume that the above ingredients are provided. In Section 4, we actually construct a particular set of worlds, together with an appropriate coercion function from worlds to assertions. In this particular case, $W \cong$ Assert holds.

The parameterization of assertions by a monoid $W$ has the interesting consequence that the following $\otimes$ operator, from Assert $\times W$ to Assert:

$$
(p \otimes w) \stackrel{\text { def }}{=} \lambda w_{0} \cdot p\left(w \circ w_{0}\right)
$$

is an action of this monoid over assertions, that is, it satisfies $p \otimes e=p$ and $(p \otimes w) \otimes w_{0}=p \otimes\left(w \circ w_{0}\right)$. These are the semantic analogues of two of the distribution axioms in Pottier's type system [4], and are also included in the logic of Section 5 . 
Healthiness conditions. The monoid of worlds and the coercion function must satisfy two further compatibility conditions. To express these, we use the abbreviation $p \circ w \stackrel{\text { def }}{=} p \otimes w * i(w)$, where $*$ denotes the pointwise lifting of the separating conjunction on $U A d m$ to Assert. The first condition is:

Condition 1. Coercions preserve $-\circ w_{0}: \forall w, w_{0} \in W . i\left(w \circ w_{0}\right)=i(w) \circ w_{0}$.

This condition lets us explain the extension of one invariant $w$ by a second invariant $w_{0}$ in terms of assertions. By unfolding the definition, we see that $i\left(w \circ w_{0}\right)$ is the assertion obtained by $*$-conjoining the assertion $i\left(w_{0}\right)$ to $i(w)$, and additionally ensuring that all computations described by the latter also preserve the invariant $w_{0}$.

The asymmetric nature of Condition 1 indicates that we cannot in general expect the monoid to be commutative. Instead, we require a weaker property: the existence of commutative pairs.

Definition 1 (Commutative pair). Let $w_{0}, w_{1}, a_{0}$ and $a_{1}$ be worlds. The pair $\left(a_{0}, a_{1}\right)$ is a commutative pair for $\left(w_{0}, w_{1}\right)$ iff (1) $w_{0} \circ a_{1}=w_{1} \circ a_{0}$, (2) $i\left(w_{0}\right) \otimes a_{1}=i\left(a_{0}\right)$ and (3) $i\left(w_{1}\right) \otimes a_{0}=i\left(a_{1}\right)$.

If $\left(a_{0}, a_{1}\right)$ is a commutative pair for $\left(w_{0}, w_{1}\right)$ then $w_{0} \circ a_{1}=$ $w_{1} \circ a_{0}$ provides an upper bound of $w_{0}$ and $w_{1}$ with respect to the extension order $\sqsubseteq$. Intuitively, we can "merge" two invariants, ensuring that all computations described in the first invariant preserve the second invariant, and vice versa.

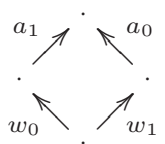

Condition 2. Every pair $\left(w_{0}, w_{1}\right)$ of worlds has a commutative pair.

Pottier's revelation lemma [4], which forms the core of his sketch of a syntactic soundness argument for his anti-frame rule, assumes the existence of commutative pairs. Commutative pairs play a similarly important role in the semantic soundness proofs below. The model described in Section 4 gives a rigorous justification for their existence.

As a consequence of Condition 1 and of the fact that $\otimes$ is a monoid action, we have the following lemma:

Lemma 2. For all $p \in$ Assert and all $w, w_{0} \in W,(p \circ w) \circ w_{0}=p \circ\left(w \circ w_{0}\right)$.

\section{Semantic Triples, Anti-frame Rule and Frame Rules}

In this section we consider the soundness of specific versions of anti-frame and frame rules, based on the semantic setting described above. For a command $c \in$ Com, let $\pi_{k}(c)$ be the command defined by $\pi_{k}(c)(h)=$ error if $c\left(\pi_{k}(h)\right)=$ error, and by $\pi_{k}(c)(h)=\pi_{k}\left(c\left(\pi_{k}(h)\right)\right)$ if $c\left(\pi_{k}(h)\right) \in$ Heap. Note that $\pi_{\infty}(c)=c$.

Definition 3. Let tri be the ternary predicate on Assert $\times$ Com $\times$ Assert such that tri $(p, c, q)$ holds iff

$$
\forall u \in U A d m . \forall h \in p(e) * u . c(h) \in \operatorname{Ad}\left(\bigcup_{w}(q \circ w)(e) * u\right),
$$

where $\operatorname{Ad}(-)$ is the admissible downward closure. 


$$
\begin{aligned}
& \text { Anti-Frame } \\
& \text { DEEP-FRAME } \\
& \frac{\models\left\{p \otimes w_{0}\right\} c\left\{q \circ w_{0}\right\}}{\models\{p\} c\{q\}} \\
& \frac{\models\{p\} c\{q\}}{\models\left\{p \circ w_{0}\right\} c\left\{q \circ w_{0}\right\}} \\
& \text { Shallow-FRAme } \\
& \overline{\{p\} c\{q\} \models\left\{p * i\left(w_{0}\right)\right\} c\left\{q * i\left(w_{0}\right)\right\}}
\end{aligned}
$$

Fig. 1. Semantic versions of a basic form of anti-frame and frame rules

This definition deserves some explanation. First, the universal quantification over $u \in U A d m$ in the definition of $\operatorname{tri}(p, c, q)$ "bakes in" the first-order frame rule, i.e., $\operatorname{tri}(p, c, q)$ is only true of commands $c$ that validate the first-order frame rule. Next, the existential quantification (union) over worlds $w \in W$ achieves the hiding of state from the post-condition of triples, as expressed by anti-frame rules. Because uniform admissible sets are not closed under arbitrary unions, we take the admissible downward closure. Technically, this makes sense because we assume commands are continuous and because we consider partial correctness only. We view the post-condition $\operatorname{Ad}\left(\bigcup_{w}(q \circ w)(e) * u\right) \subseteq$ Heap as a subset of Heap $\oplus\{\text { error }\}_{\perp}$ in the evident way. In particular, this means that $\operatorname{tri}(p, c, q)$ is only true of commands $c$ that do not fault for states in the pre-condition. This definition does not "bake in" monotonicity w.r.t. invariants (worlds): $p$ and $q \circ w$ are "closed" just by applying them to the empty world. This is rectified in the following definition of validity:

Definition 4 (Validity). A (semantic) triple $\{p\} c\{q\}$ holds with respect to $w$ and $k \in \mathbb{N}_{\infty}$, which we write $\left.w\right|_{k}\{p\} c\{q\}$, iff $\operatorname{tri}\left(p \circ\left(w \circ w_{0}\right), \pi_{k}(c), q \circ\left(w \circ w_{0}\right)\right)$ holds for all $w_{0} \in W$. We sometimes omit the index when $k=\infty$.

As a consequence of the quantification over worlds $w_{0}$ in this definition, the validity of a triple is monotonic: if $w \models_{k}\{p\} c\{q\}$ and $w \sqsubseteq w^{\prime}$ then $\left.w^{\prime}\right|_{k}\{p\} c\{q\}$. The approximate validity (i.e., the case where $k \neq \infty$ ) is used when considering nested triples. Recall that assertions are the contractive monotone functions from $W$ to $U A d m 5$ Because nested triples will be interpreted as elements in Assert, they must be contractive. The approximations will allow us to satisfy this requirement. We write $\models\{p\} c\{q\}$ to mean that $w \models_{k}\{p\} c\{q\}$ for all $k, w$. Also, we write $\{p\} c\{q\} \models\left\{p^{\prime}\right\} c^{\prime}\left\{d^{\prime}\right\}$ to mean that $w \models_{k}\{p\} c\{q\} \Rightarrow w \models_{k}\left\{p^{\prime}\right\} c^{\prime}\left\{q^{\prime}\right\}$ holds for all $w, k$.

We are now ready to describe semantic versions of examples of anti-frame and frame rules and to prove their soundness. The semantic rules are given in Fig. 1, where the first two rules should be understood as the implication from the premise to the conclusion.

Our first lemma is a consequence of the monotonicity of assertions.

Lemma 5. For all $w$, we have that (1) $\operatorname{tri}(p \otimes w, c, q) \Rightarrow \operatorname{tri}(p, c, q)$ and (2) $\operatorname{tri}(p, c, q \circ w) \Rightarrow \operatorname{tri}(p, c, q)$.

Proof. For the first implication, suppose that $u \in U A d m$ and $h \in p(e) * u$. By the monotonicity of $p$ and by $e \sqsubseteq w$ we obtain $p(e) \subseteq p(w)=p(w \circ e)=(p \otimes w)(e)$.

\footnotetext{
${ }^{5}$ More precisely, they are the non-expansive monotone functions from $\frac{1}{2} \cdot W$ to $U A d m$.
} 
Therefore, $h \in(p \otimes w)(e) * u$. The result now follows from the assumption that $\operatorname{tri}(p \otimes w, c, q)$ holds.

For the second implication, suppose again that $u \in U A d m$ and $h \in p(e) * u$. We must show that $c(h) \in \operatorname{Ad}\left(\bigcup_{w^{\prime}}\left(q \circ w^{\prime}\right)(e) * u\right)$. From the assumption that $\operatorname{tri}(p, c, q \circ w)$ holds we obtain $c(h) \in \operatorname{Ad}\left(\bigcup_{w^{\prime \prime}}\left((q \circ w) \circ w^{\prime \prime}\right)(e) * u\right)$. By Lemma2,

$$
\bigcup_{w^{\prime \prime}}\left((q \circ w) \circ w^{\prime \prime}\right)(e)=\bigcup_{w^{\prime \prime}}\left(q \circ\left(w \circ w^{\prime \prime}\right)\right)(e) \subseteq \bigcup_{w^{\prime}}\left(q \circ w^{\prime}\right)(e) .
$$

The result then follows from the monotonicity of $*$ and the monotonicity of the closure operation $\operatorname{Ad}(\cdot)$.

The next lemma amounts to gluing two commutative pair diagrams together (along $a_{0}$ there). Its proof involves the associativity of $\circ$ as well as Condition 1 ,

Lemma 6. If $\left(a_{0}, a_{1}\right)$ is a commutative pair for $\left(w_{0}, w_{1}\right)$ and $\left(b_{0}, a_{2}\right)$ is a commutative pair for $\left(a_{0}, w_{2}\right)$ then $\left(b_{0}, a_{1} \circ a_{2}\right)$ is a commutative pair for $\left(w_{0}, w_{1} \circ w_{2}\right)$.

The following proposition combines Lemmas [5] and 6] and relates the validity of two triples in our anti-frame rule.

Proposition 7. For all worlds $\left(w_{0}, w\right)$, if $\left(a_{0}, a\right)$ is a commutative pair for $\left(w_{0}, w\right)$, then $a \models_{k}\left\{p \otimes w_{0}\right\} c\left\{q \circ w_{0}\right\}$ implies $w \models_{k}\{p\} c\{q\}$.

Proof. We need to show that $w=_{k}\{p\} c\{q\}$, which by definition means that for all $w_{1}$, letting $w_{2}=w \circ w_{1}$ and $d=\pi_{k}(c)$,

$$
\operatorname{tri}\left(p \circ w_{2}, d, q \circ w_{2}\right)
$$

By Condition 2, there exists a commutative pair $\left(b_{0}, a_{1}\right)$ for $\left(a_{0}, w_{1}\right)$. Let $b_{2}=$ $a \circ a_{1}$. By Lemma 6, $\left(b_{0}, b_{2}\right)$ is a commutative pair for $\left(w_{0}, w \circ w_{1}\right)=\left(w_{0}, w_{2}\right)$. In particular, we have $w_{0} \circ b_{2}=w_{2} \circ b_{0}$ and $i\left(b_{2}\right)=i\left(w_{2}\right) \otimes b_{0}$. The assumed triple implies tri $\left(\left(p \otimes w_{0}\right) \circ b_{2}, d,\left(q \circ w_{0}\right) \circ b_{2}\right)$. Thus,

$$
\operatorname{tri}\left(\left(p \circ w_{2}\right) \otimes b_{0}, d,\left(q \circ w_{2}\right) \circ b_{0}\right)
$$

follows, using the following equalities:

$$
\begin{aligned}
\left(p \otimes w_{0}\right) \circ b_{2} & =p \otimes\left(w_{0} \circ b_{2}\right) * i\left(b_{2}\right)=p \otimes\left(w_{2} \circ b_{0}\right) * i\left(w_{2}\right) \otimes b_{0} \\
& =\left(p \otimes w_{2}\right) \otimes b_{0} * i\left(w_{2}\right) \otimes b_{0}=\left(p \otimes w_{2} * i\left(w_{2}\right)\right) \otimes b_{0} \\
& =\left(p \circ w_{2}\right) \otimes b_{0}, \\
\left(q \circ w_{0}\right) \circ b_{2} & =q \circ\left(w_{0} \circ b_{2}\right)=q \circ\left(w_{2} \circ b_{0}\right)=\left(q \circ w_{2}\right) \circ b_{0} .
\end{aligned}
$$

From (4), we derive the desired triple (3) as shown below:

$$
\begin{aligned}
\operatorname{tri}\left(\left(p \circ w_{2}\right) \otimes b_{0}, d,\left(q \circ w_{2}\right) \circ b_{0}\right) & \Rightarrow \operatorname{tri}\left(p \circ w_{2}, d,\left(q \circ w_{2}\right) \circ b_{0}\right) \\
& \Rightarrow \operatorname{tri}\left(p \circ w_{2}, d, q \circ w_{2}\right) .
\end{aligned}
$$

Both implications hold because of Lemma 5 
Corollary 8 (Anti-frame rule). The anti-frame rule in Fig. 1 is sound.

Proof. Pick $w, k$. Let $p, c, q, w_{0}$ be as in the anti-frame rule in Fig. 1. We must prove that $w \models_{k}\{p\} c\{q\}$. By Condition 2, there exists a commutative pair $\left(a_{0}, a\right)$ for $\left(w_{0}, w\right)$. By assumption, we have $\models\left\{p \otimes w_{0}\right\} c\left\{q \circ w_{0}\right\}$, so, in particular, $a \models k$ $\left\{p \otimes w_{0}\right\} c\left\{q \circ w_{0}\right\}$. By Proposition 7, this implies $w \models_{k}\{p\} c\{q\}$, as desired.

Next, we move on to the soundness proof of the two frame rules in Fig. 1,

Lemma 9. The following equivalence, which expresses a distribution axiom [9], holds: $w_{0} \circ w \models_{k}\{p\} c\{q\}$ iff $w \models_{k}\left\{p \circ w_{0}\right\} c\left\{q \circ w_{0}\right\}$.

Proof. Pick $w_{1}$. Let $w_{1}^{\prime}=w \circ w_{1}$. By Definition 4 and the associativity of $\circ$, it suffices to prove the equivalence of $\operatorname{tri}\left(p \circ\left(w_{0} \circ w_{1}^{\prime}\right), \pi_{k}(c), q \circ\left(w_{0} \circ w_{1}^{\prime}\right)\right)$ and $\operatorname{tri}\left(\left(p \circ w_{0}\right) \circ w_{1}^{\prime}, \pi_{k}(c),\left(q \circ w_{0}\right) \circ w_{1}^{\prime}\right)$. This equivalence follows from Lemma 2

Corollary 10 (Frame rules). The frame rules in Fig. 1] are sound.

Proof. The soundness of the deep frame rule follows from Lemma 9. The shallow rule is sound thanks to the universal quantification over $u$ in Definition 3 ,

\section{A Concrete Model with Recursively Defined Worlds}

In this section, we consider a concrete instance of the general framework described in Sections 2 and 3 where $W$ is isomorphic to Assert. The Assert $\rightarrow W$ direction of this isomorphism means that all assertions can be used as hidden invariants. This lets us define a semantic model of the program logic that is presented next (Section [5). The heap model in this particular case is given by the following recursively defined cpos:

$$
\text { Heap }=\operatorname{Rec}(\mathrm{Val}) \quad \mathrm{Val}=\mathrm{In} \mathrm{t}_{\perp} \oplus \mathrm{Com}_{\perp} \quad \mathrm{Com}=\mathrm{Heap} \multimap \mathrm{Heap} \oplus\{\text { error }\}_{\perp}
$$

where $\operatorname{Rec}(\mathrm{Val})$ denotes records with entries in $\mathrm{Val}$ labelled by positive natural number 6 . These labels serve as addresses or locations. The partial operation $h \cdot h^{\prime}$ combines two heaps $h$ and $h^{\prime}$ (i.e., takes the union) whenever the domains of $h$ and $h^{\prime}$ are disjoint. When $h=\perp$ or $h^{\prime}=\perp, h \cdot h^{\prime}$ is $\perp$. The empty record provides a unit for heap combination, thus there is also a unit for the separating conjunction on $U A d m$. Finally, the solution of (5) in the category $\mathbf{C p p o}_{\perp}$ of pointed cpos and strict continuous functions comes equipped with a family of projections $\pi_{k}$ that satisfy the requirements of Section 2.

The key result of this section is the following theorem:

Theorem 11. There exists a monoid $(W, e, \circ)$, where $W$ is an object in CBUlt with an isomorphism $\iota$ from $W$ to $\left(\frac{1}{2} \cdot W\right) \rightarrow_{m} U A d m$. The operation o satisfies

$$
\forall w_{1}, w_{2}, w \in W . \iota\left(w_{1} \circ w_{2}\right)(w)=\iota\left(w_{1}\right)\left(w_{2} \circ w\right) * \iota\left(w_{2}\right)(w) .
$$

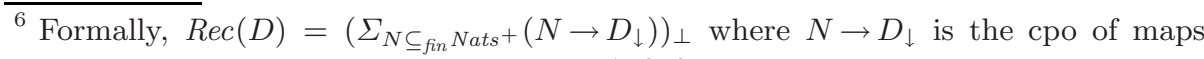
from the finite address set $N$ to $D_{\downarrow}=D \backslash\{\perp\}$ of non-bottom elements of $D$. 
The equation in this theorem is just Condition 11, where the coercion $i$ is taken to be the isomorphism $\iota$.

Construction of the worlds $W$ in Theorem [1], In previous work [9] we gave a model of a separation logic with nested triples and higher-order frame rules, but no anti-frame rule. For this model we needed a solution $W^{\prime}$ to the following domain equation:7

$$
W^{\prime} \cong\left(\frac{1}{2} \cdot W^{\prime}\right) \rightarrow U A d m
$$

Note that (6) is almost the same domain equation as described in Theorem 11 above, except that there is no restriction to monotonic functions in the function space on the right. One can use a general existence theorem [12, 13] to obtain a solution $W^{\prime}$ for (6) in the category CBUlt. In a second step, using the complete metric on $W^{\prime}$, one can then define a monoid operation $\circ$ that satisfies the equation stated in Theorem 11.

In the present setup, this two-step approach to constructing a solution $W \cong$ Assert and a monoid operation o cannot be applied, however, because of the added monotonicity requirement in Theorem 11] since the order on $W$ is defined in terms of the operation $\circ$, one needs $\circ$ already in order to express this equation, i.e., it appears necessary to define the operation o at the same time as $W$. Thus we construct $W \cong\left(\frac{1}{2} \cdot W\right) \rightarrow_{m} U A d m$ explicitly, as (inverse) limit

$$
W=\left\{x \in \prod_{k \geq 0} W_{k} \mid \forall k \geq 0 . \iota_{k}^{\circ}\left(x_{k+1}\right)=x_{k}\right\}
$$

of a sequence of "approximations" $W_{k}$ of $W$,

$$
W_{0} \underset{\iota_{0}^{\circ}}{\stackrel{\iota_{0}}{\rightleftarrows}} W_{1} \underset{\iota_{1}^{\circ}}{\stackrel{\iota_{1}}{\rightleftarrows}} W_{2} \underset{\iota_{2}^{\circ}}{\stackrel{\iota_{2}}{\rightleftarrows}} \cdots \frac{\iota_{k}}{\underset{\iota_{k}^{\circ}}{\rightleftarrows}} W_{k+1} \underset{\iota_{k+1}^{\circ}}{\stackrel{\iota_{k+1}}{\rightleftarrows}} \cdots
$$

Each $W_{k}$ is a complete 1-bounded ultrametric space equipped with a nonexpansive operation $\circ_{k}: W_{k} \times W_{k} \rightarrow W_{k}$ and a preorder $\sqsubseteq_{k}$, so that $W_{k+1}=$ $\left(\frac{1}{2} \cdot W_{k}\right) \rightarrow_{m} U A d m$ are the non-expansive and monotone functions with respect to $\sqsubseteq_{k}$. The maps $\iota_{k}$ and $\iota_{k}^{\circ}$ are given by $\iota_{k+1}(w)=\pi_{k+2} \circ w \circ \iota_{k}^{\circ}$ and $\iota_{k+1}^{\circ}(w)=\pi_{k+1} \circ w \circ \iota_{k}$. The diagram (7) forms a Cauchy tower [13], in that $\sup _{w} d_{k+1}\left(w,\left(\iota_{k} \circ \iota_{k}^{\circ}\right)(w)\right)$ and $\sup _{w} d_{k}\left(w,\left(\iota_{k}^{\circ} \circ \iota_{k}\right)(w)\right)$ become arbitrarily small as $k$ increases. The operation $\circ_{k+1}$ is defined in terms of $\circ_{k}$ and $\iota_{k}^{\circ}$ :

$$
\left(w_{1} \circ_{k+1} w_{2}\right)(w) \stackrel{\text { def }}{=} w_{1}\left(\iota_{k}^{\circ}\left(w_{2}\right) \circ_{k} w\right) * w_{2}(w) .
$$

One technical inconvenience is that the $\circ_{k}$ 's are not associative. However, associativity holds "up to approximation $k, " d_{k}\left(\left(x \circ_{k} y\right) \circ_{k} z, x \circ_{k}\left(y \circ_{k} z\right)\right) \leq 2^{-k}$, which yields associativity "in the limit" and thus a monoid structure on $W$ by

$$
\left(x_{k}\right)_{k \geq 0} \circ\left(y_{k}\right)_{k \geq 0} \stackrel{\text { def }}{=}\left(\lim _{j>k} \iota_{k}^{\circ}\left(\ldots\left(\iota_{j-1}^{\circ}\left(x_{j} \circ_{j} y_{j}\right)\right)\right)_{k \geq 0} .\right.
$$

\footnotetext{
7 Technically, we solved a different equation $W^{\prime} \cong \frac{1}{2}\left(W^{\prime} \rightarrow U A d m\right)$. This difference is
} insignificant, since the solution of one equation leads to that of the other equation. 
To finish the proof of Theorem 11 one shows that $\iota(w) \stackrel{\text { def }}{=} \lim _{k}\left(\lambda w^{\prime} \cdot w_{k+1}\left(w_{k}^{\prime}\right)\right)$ establishes an isomorphism $\iota$ between $W$ and $\left(\frac{1}{2} \cdot W\right) \rightarrow_{m} U A d m$, which satisfies $\iota\left(w_{1} \circ w_{2}\right)=\iota\left(w_{1}\right) \otimes w_{2} * \iota\left(w_{2}\right)$ for $(p \otimes w)=\lambda w^{\prime} \cdot p\left(w \circ w^{\prime}\right)$

The details of the proof are given in the full version of this paper [14]. Existence of commutative pairs. To show that $W$ forms an instance of our semantic framework, we also need to prove the existence of commutative pairs. Given a pair $\left(w_{0}, w_{1}\right)$ of worlds, we construct a commutative pair using properties of $\otimes$ and the coercion $\iota$. Since the monoid operation $\otimes$ is contractive in its second argument, so is the function $f\left(a_{0}, a_{1}\right)=\left(\iota^{-1}\left(\iota\left(w_{0}\right) \otimes a_{1}\right), \iota^{-1}\left(\iota\left(w_{1}\right) \otimes a_{0}\right)\right)$ on $W \times W$. By the Banach fixed point theorem, there exists a unique pair $\left(a_{0}, a_{1}\right)=f\left(a_{0}, a_{1}\right)$. Thus $\iota\left(a_{0}\right)=\iota\left(w_{0}\right) \otimes a_{1}$ and $\iota\left(a_{1}\right)=\iota\left(w_{1}\right) \otimes a_{0}$. Since $\iota$ is injective, we can prove the remaining $w_{0} \circ a_{1}=w_{1} \circ a_{0}$ as follows. For all $w \in W$,

$$
\begin{aligned}
\iota\left(w_{0} \circ a_{1}\right)(w) & =\left(\iota\left(w_{0}\right) \otimes a_{1}\right)(w) * \iota\left(a_{1}\right)(w) & & (\text { by Theorem 11 and def. of } \otimes) \\
& =\iota\left(a_{0}\right)(w) *\left(\iota\left(w_{1}\right) \otimes a_{0}\right)(w) & & \text { (by the above properties of } \left.a_{0}, a_{1}\right) \\
& =\iota\left(a_{0}\right)(w) * \iota\left(w_{1}\right)\left(a_{0} \circ w\right) & & \text { (by def. of } \otimes) \\
& =\iota\left(w_{1} \circ a_{0}\right)(w) & & \text { (by Theorem 11). }
\end{aligned}
$$

Theorem 12. The monoid $(W, e, \circ)$ in Theorem 11 and the isomorphism $\iota$ : $W \rightarrow$ Assert form an instance of the framework in Section 2 .

\section{Program Logic}

We now give one application of our semantic development. We present a program logic for higher-order store, which includes anti-frame and frame rules. Using the results of Sections 3 and 4, we define the semantics of the logic and prove its soundness.

Programming language. Fig. 2 gives the syntax of a small imperative programming language equipped with operations for stored code and heap manipulation. The expressions in the language are integer expressions, variables, and the quote expression ' $C$ ' for representing an unevaluated command $C$. The integer or code value denoted by expression $e_{1}$ is stored in a heap cell $e_{0}$ using $\left[e_{0}\right]:=e_{1}$, and this stored value is later looked up and bound to the variable $y$ by let $y=\left[e_{0}\right]$ in $D$. In the case that the value stored in cell $e_{0}$ is code ' $C$ ', we can run (or "evaluate") this code by executing eval $\left[e_{0}\right]$. As in ML, all variables $x, y, z$ are immutable. The language does not include while loops: they can be expressed by stored code (using Landin's knot). The interpretation of commands in the cpo Com of (5]) is straightforward [9]. The interpretation of the quote operation, ' $C$ ', uses the injection of Com into $\mathrm{Val}$ in Section 4.

Assertions and distribution axioms. As in previous work 9], our assertion language is first-order intuitionistic logic, extended with the separating connectives e, $*$, and the points-to predicate $\mapsto[5]$. The syntax of assertions appears in Fig. 2] The standard connectives are omitted.

The most distinguishing features of the assertion language are Hoare triples $\{P\} e\{Q\}$ and invariant extensions $P \otimes Q$. The fact that a triple is an assertion 


\begin{tabular}{|c|c|}
\hline$e \in \operatorname{Exp}::=-1|1| e_{1}+e_{2}|\ldots| x \mid{ }^{\prime} C$ ' & integer expression, variable, quote \\
\hline $\begin{aligned} C \in C o m::= & {\left[e_{1}\right]:=e_{2} \mid \text { let } y=[e] \text { in } C \mid \text { eval }[e] } \\
\mid \begin{array}{l}\text { let } x=\text { new }\left(e_{1}, \ldots, e_{n}\right) \text { in } C \mid \text { free } e \\
\mid\end{array} & \text { skip }\left|C_{1} ; C_{2}\right| \text { if }\left(e_{1}=e_{2}\right) C_{1} C_{2}\end{aligned}$ & $\begin{array}{l}\text { assignment, lookup, unquote } \\
\text { allocation, disposal } \\
\text { no op, sequencing, conditional }\end{array}$ \\
\hline $\begin{aligned} & P, Q \in A s s n::= e_{1} \mapsto e_{2}|\mathrm{e}| P * Q \\
& \mid \begin{array}{l} \\
\{P\} e\{Q\} \mid P \otimes Q \\
X|\mu X . P| \ldots\end{array}\end{aligned}$ & $\begin{array}{l}\text { separating connectives } \\
\text { Hoare triple, invariant extension } \\
\text { assertion variable, recursion }\end{array}$ \\
\hline
\end{tabular}

Fig. 2. Syntax of expressions, commands and assertions

$$
\begin{aligned}
& \{P\} e\{Q\} \otimes R \Leftrightarrow\{P \circ R\} e\{Q \circ R\} \\
& (\kappa x . P) \otimes R \Leftrightarrow \kappa x .(P \otimes R) \\
& (P \otimes R) \otimes R^{\prime} \Leftrightarrow P \otimes\left(R \circ R^{\prime}\right) \\
& (P \oplus Q) \otimes R \Leftrightarrow(P \otimes R) \oplus(Q \otimes R) \\
& P \otimes R \Leftrightarrow P \\
& (\mu X . P) \otimes R \Leftrightarrow \mu X .(P \otimes R) \\
& (\kappa \in\{\forall, \exists\}, x \notin f v(R)) \\
& (\oplus \in\{\Rightarrow, \wedge, \vee, *\})
\end{aligned}
$$

Fig. 3. Axioms for distributing $-\otimes R$

means that triples can be nested. Intuitively, the assertion $P \otimes Q$ denotes a version of $P$ where every (possibly deeply nested) triple receives a copy of $Q$ as an extra *-conjunct in its pre- and post-conditions. More precisely, the behaviour of the $\otimes$ operator is described by the axioms in Fig. 3, which let us distribute $\otimes$ through all the constructs of the assertion language. These axioms use the abbreviation $Q \circ R$ for $(Q \otimes R) * R$.

Assertions include assertion variables $X \in \mathcal{X}$, and can be recursively defined: the construct $\mu X . P$ binds $X$ in $P$ and satisfies the axiom $\mu X . P \Leftrightarrow P[X:=$ $\mu X . P]$. Not every recursive assertion is permitted: for $\mu X . P$ to be well-formed, we require that $P$ be formally contractive in $X$. In short, this means that every free occurrence of $X$ within $P$ must lie either within a triple or within the second argument of a $\otimes$ construct. (We omit the straightforward inductive definition of formal contractiveness.) Semantically, this requirement ensures that $\mu X . P$ is well-defined as the unique fixed point of $P$, viewed as a function of $X$. In particular, all assertions of the form $\mu X . P \otimes X$, where $X$ does not appear in $P$, are formally contractive. Pottier's applications of the anti-frame rule [4] make extensive use of assertions of this form.

The interpretation of assertions uses $W$ in Section 4 , Given such $W$ and an environment $\eta$ that maps variables $x$ to values $\eta(x) \in V a l$, we interpret an assertion $P$ as a non-expansive function $\llbracket P \rrbracket_{\eta}:$ Assert ${ }^{\mathcal{X}} \rightarrow$ Assert. The uniform admissible sets in $U A d m$, partially ordered by inclusion, form a complete Heyting algebra with a monotone commutative monoid. The domain Assert, ordered pointwise, inherits this structure (see Appendix B of the full version of this 


$$
\begin{gathered}
\llbracket P \otimes R \rrbracket_{\eta, \xi}=\llbracket P \rrbracket_{\eta, \xi} \otimes \iota^{-1}\left(\llbracket R \rrbracket_{\eta, \xi}\right) \quad \quad \llbracket \mu X . P \rrbracket_{\eta, \xi}=f i x\left(\lambda q . \llbracket P \rrbracket_{\eta, \xi[X:=q]}\right) \\
\llbracket\{P\}^{\prime} C^{\prime}\{Q\} \rrbracket_{\eta, \xi}=\lambda w \cdot\left\{h \mid r n k(h)>0 \Rightarrow w \models_{r n k(h)-1}\left\{\llbracket P \rrbracket_{\eta, \xi}\right\} \llbracket C \rrbracket_{\eta}\left\{\llbracket Q \rrbracket_{\eta, \xi}\right\}\right\}
\end{gathered}
$$

Fig. 4. Interpretation of assertions

$\begin{array}{lll}\text { Anti-Frame } & \text { DeEP-Frame } & \text { Shallow-Frame } \\ \frac{\Gamma ; \Xi \vdash\{P \otimes R\} e\{Q \circ R\}}{\Gamma ; \Xi \vdash\{P\} e\{Q\}} \frac{\Gamma ; \Xi \vdash\{P\} e\{Q\}}{\Gamma ; \Xi \vdash\{P \circ R\} e\{Q \circ R\}} & & \\ ; \Xi \vdash\{P\} e\{Q\} \Rightarrow\{P * R\} e\{Q * R\}\end{array}$

Fig. 5. Proof rules from separation logic

paper [14]). This is used to interpret the intuitionistic first-order fragment, * and e of the assertion language. Fig. 4 shows three of the remaining cases. First, via the isomorphism $\iota^{-1}$, we can turn any assertion $r \in$ Assert into an invariant $\iota^{-1}(r) \in W$ and thus interpret the invariant extension $P \otimes R$. Next, because $P$ must be formally contractive in $X$, the map $q \mapsto \llbracket P \rrbracket_{\eta, \xi[X:=q]}$ on Assert is contractive in the metric sense: thus, by the Banach fixed point theorem, it has a unique fixed point. Finally, the interpretation of nested triples is in terms of semantic triples, and uses approximate validity to ensure non-expansiveness.

Proof rules. The logic derives judgements of the form $\Gamma ; \Xi \vdash P$, where $P$ is an assertion, and $\Gamma$ and $\Xi$ respectively bind variables and assertion variables. For instance, to prove that command $C$ stores at cell 1 some code that writes 0 into cell 10, one would need to derive $\Gamma ; \Xi \vdash\left\{1 \mapsto_{-}\right\}^{\prime} C^{\prime}\left\{1 \mapsto\left\{10 \mapsto{ }_{-}\right\}-\{10 \mapsto 0\}\right\}$.

The logic includes the standard proof rules for intuitionistic logic and the logic of bunched implications [16] as well as standard separation logic proof rules [9]. We do not repeat these rules here. Fig. 5 shows a version of the anti-frame rule and two versions of the frame rule: the deep frame rule (expressed in combination with the distribution axioms) and the first-order shallow frame rule (which takes the form of an axiom).

Theorem 13 (Soundness). The interpretation of assertions is well-defined, and validates the distribution axioms of Fig. 3 and the inference rules of Fig. 5 .

\section{Conclusion and Future Work}

We have presented a semantic framework for studying the soundness of antiframe and frame rules for languages with higher-order store. Moreover, we have presented a concrete instance of the framework, and used it to give the first rigorous proof of soundness of separation logic with anti-frame and frame rules for a language with higher-order store. 
We are aware of other instantiations of the semantic framework, which can be used to show the soundness of stronger variants of the anti-frame and frame rules, provided the universe $W$ of invariants is restricted. For space reasons, we have not included those instantiations in this extended abstract; they appear in the full version of the paper 14].

Future work includes lifting the results in this paper to Pottier's type-andcapability system as well as extending our soundness results to generalized versions of the anti-frame and frame rules where invariants evolve in more sophisticated ways over time [17, 18].

Acknowledgments. We would like to thank Kristian Støvring and Jacob Thamsborg for helpful discussions. Partial support has been provided by FNU project 272-07-0305 "Modular reasoning about software" and EPSRC.

\section{References}

1. Parkinson, M., Bierman, G.: Separation logic and abstraction. In: POPL, pp. 247$258(2005)$

2. Biering, B., Birkedal, L., Torp-Smith, N.: BI-hyperdoctrines, higher-order separation logic, and abstraction. TOPLAS 29(5) (2007)

3. Parkinson, M., Bierman, G.: Separation logic, abstraction and inheritance. In: POPL, pp. 75-86 (2008)

4. Pottier, F.: Hiding local state in direct style: a higher-order anti-frame rule. In: LICS, pp. 331-340 (2008)

5. Reynolds, J.C.: Separation logic: A logic for shared mutable data structures. In: LICS, pp. 55-74 (2002)

6. O'Hearn, P.W., Yang, H., Reynolds, J.C.: Separation and information hiding. In: POPL, pp. 268-280 (2004)

7. Birkedal, L., Torp-Smith, N., Yang, H.: Semantics of separation-logic typing and higher-order frame rules for Algol-like languages. LMCS 2(5:1) (2006)

8. Birkedal, L., Reus, B., Schwinghammer, J., Yang, H.: A simple model of separation logic for higher-order store. In: Aceto, L., Damgård, I., Goldberg, L.A., Halldórsson, M.M., Ingólfsdóttir, A., Walukiewicz, I. (eds.) ICALP 2008, Part II. LNCS, vol. 5126, pp. 348-360. Springer, Heidelberg (2008)

9. Schwinghammer, J., Birkedal, L., Reus, B., Yang, H.: Nested Hoare triples and frame rules for higher-order store. In: CSL, pp. 440-454 (2009)

10. Pottier, F.: Three comments on the anti-frame rule (July 2009) (unpublished note)

11. Levy, P.B.: Possible world semantics for general storage in call-by-value. In: CSL, pp. 232-246 (2002)

12. Rutten, J.J.M.M.: Elements of generalized ultrametric domain theory. TCS 170(12), 349-381 (1996)

13. Birkedal, L., Støvring, K., Thamsborg, J.: The category-theoretic solution of recursive metric-space equations. Technical Report ITU-2009-119, IT University of Copenhagen (2009)

14. Schwinghammer, J., Yang, H., Birkedal, L., Pottier, F., Reus, B.: A semantic foundation for hidden state (December 2009), http://www.dcs.qmul.ac.uk/ hyang/paper/fossacs10-full.pdf 
15. Streicher, T.: Domain-theoretic Foundations of Functional Programming. World Scientific, Singapore (2006)

16. O'Hearn, P.W., Pym, D.J.: The logic of bunched implications. Bulletin of Symbolic Logic 5(2), 215-244 (1999)

17. Pilkiewicz, A., Pottier, F.: The essence of monotonic state (October 2009) (submitted)

18. Pottier, F.: Generalizing the higher-order frame and anti-frame rules (July 2009) (unpublished note) 